0.1792 g Sbst.: $0.5271 \mathrm{~g} \mathrm{CO}_{8}, 0.1144 \mathrm{~g} \mathrm{H}_{2} \mathrm{O}$.

$$
\begin{aligned}
& \mathrm{C}_{17} \mathrm{H}_{18} \mathrm{O}_{2} \text {. Ber. C } 80.3 \text {, H 7.1. } \\
& \text { Gef. \$ } 80.22 \text {, 7.14. }
\end{aligned}
$$

Wir hoffen, bald zeigen zu können, daß die Einwirkung homologer Phenole auf Methylcumarsäuredibromid in noch anderer und ganz unerwarteter Weise verläuft.

Rostock, Juni 1911.

\title{
244. R. Stoermer: Über Phenyl-Wanderungen bei der Synthese phenylierter Cumarone. (Zur Kenntnis des Phosphortribromids als Reduktionsmittel. III.)
}

[Mitteilung aus den Chemischen Institut der Universität Rostock.]

(Eingegangen am 13. Juni 1911.)

In früheren Abhandlungen über die Reduktionswirkung des Phosphortribromids ${ }^{1}$ ) ist ron mir $\mathrm{z}$. T. gemeinsam mit $\mathrm{O}$. Kippe und 0 . Martinsen gezeigt worden, daß viele Verbindungen mit der Atomgruppierung $\mathrm{CO} . \mathrm{CH}_{2}$ bezw. NH.CO beim Erbitzen mit dem genannten Agens großenteils sehr leicht zu sauerstofffreien Körpern reduziert werden können, die den Komplex $\mathrm{CH}: \mathrm{CH}$ bezw. N:CH besitzen, ein Verfabren, das sich ganz besonders zur bequemen Darstellung beliebiger Pyrazole aus Pyrazolonen ausgestalten ließ. Aber auch für gewissse andere Körperklassen hat sich die Methode als ausgezeichnet brauchbar erwiesen, so vor allem für die Reduktion gewisser aromatischer $\gamma$-Lactone zu Cumaron-Derivaten, die s) sebr leicht zugänglich sind, da die betreffenden Lactone, z. B. das - Oxy-diphenylessigsäure-lacton und alle seine Abkömmlinge sach den Arbeiten Bistrzyckis und seiner Schüler ${ }^{2}$ ) in beliebiger Menge darstellbare Ausgangsmaterialien sind.

Die Umsetzung geht hier in der Weise vor sich, daß bei Anwendung von Phosphortribromid unter gewöhnlichem Druck bei wöherer Temperatur in ziemlich guter Ausbeute 2-phenylierte $\mathrm{Cu}$ marone entstehen, während oberbalb $200^{\circ}$ im geschlossenen Rohr neben den genannten Verbindungen auch solche Cumarone sich bilden, die das Phenyl in der 1-Stellung enthalten. Onter Beibehaltung der

1) 1. Abhandlung, B. 36, 3986 [1908]; 2. Abhandlung, A. 352, 322 [1907].

3) Bistrzycki und Flatau, B. 30, 127 [1897]; Bistrzycki und Simonis, B. 31, 2812 [1898]; Cramer, B. 31, 2813 [1898]; Simonis, B. 31, 2821 [1898] u. a. 
Annahme eines labilen $Z$ wischenproduktes würde die Reaktion nacb dem Schema verlaufen:

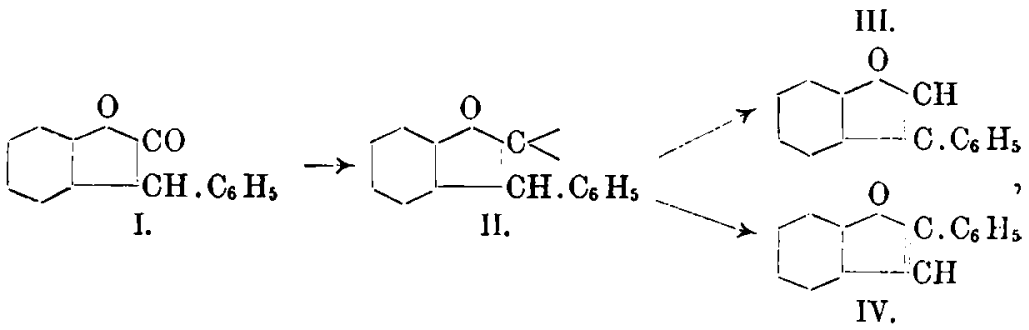

und gerade die Entstehung der beiden Isomeren diente als Stütze für die Auffassung, daß intermediär nicht ein gebromtes Phenylcumaron, sondern ein nicht selbständig existenzfähiges $Z$ wischenprodukt entstanden war, von dem aus die Bildung der beiden Phenylcumarone ohne weiteres verständlich erschien.

Wäbrend nun allerdings bei den Pyrazolonen die Bildung von Brompyrazolen bei der Einwirkung von Phosphorbromiden bei niederer Temperatur sich leicht nachweisen ließ '), ist etwas $\ddot{A}$ hnliches bei den genannten Lactonen nicht der Fall: auch bei niedriger Temperatur konnten niemals Bromphenyl-cumarone erhalten werden, und diese letzteren, auf anderem Wege dargestellt,werden durch Erhitzen mit Pbosphortribromid nicht verändert. Die Versuche haben also erwiesen, da $\$$ man nicht umbin kann, in dieser Gruppe der Lactone, die anscheinend nur sehr schwer dazu neigen, in Enolformen überzugehell, die vorübergehende Existen $z$ von labilen $Z$ wischenformen anzunehmen. Auch eine sekundäre Umlagerung des primär entstandenen 2-Phevjlcumarons erscheint ausgeschlossen, denn zablreiche Versuche ${ }^{2}$ ), diesen Körper durch Erhitzen mit Phosphortribromid bei den verscbiedensten Temperaturen in 1-Phenylcumaron umzulagern, sind ohne jedes Ergebnis geblieben, vielmehr wurde das unveränderte Material quantitativ zurückgewonnen. Auffällig erscheint nur, daß es nicht gelang, Oxydiphenyl-bromessigsäurelacton auf dem gleichen Wege in Brompinenylcumaron umzulagern:

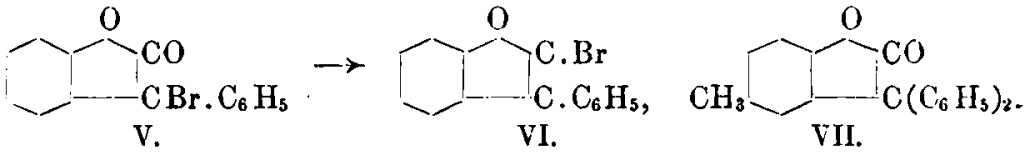

1) Michaelis und Behn, B. 33, 2603 [1900]; Stoermer und Martinseu, loc. cit.

2) Vergl. Claus Decker, Zur Kenntnis phenylierter Cumarone, Dissertation, Rostock 1907. 
Wenigstens konnten die sehr geringen Mengen des gewonnenen brombaltigen Oles nicht mit Sicherheit mit Brom-phenyl-cumaron identifiziert werden. Auch auf das Kresyl-diphenylessigsäure-lacton(VII) war Phosphortribromid ohne jede Einwirkung, und die erwartete Umwandlung in 1.2-Diphenyl-4-methylcumaron war nicht eingetreten.

Die Neigung zur Wanderung des Phenyls in die 1-Stellung ist bei der angeführten Reaktion nur sehr gering und wird auch durch Einführung von geeigneten Substituenten in den Benzolkern nicht vergrößert, sondern im Gegenteil nur noch verringert, so daß bei den in der 4-Stellung balogenierten Lactonen überbaupt keine Radikalverschiebung mehr eintritt, sondern nur die Bildung von 2-Phenylcumaronen (in ganz guter Ausheute) beobachtet werden kann ${ }^{1}$ ). Das Mißlingen dieser Versuche ist darayf zurückzuführen, daß zur Umlagerung ziemlich hohe Temperaturen (bis $250^{\circ}$ ) erforderlich sind, bei denen aber die anderweitigen Zersetzungen so überwiegen können, daß es zur Bildung von 1-Phenylcumaronen nicht mehr kommt. Eine leidlich gute Ausbeute an einem solchen erhält man überhaupt nur bei dem nicht substituierten Lacton.

Um die Konstitution der nur in so kleiner Menge erhältlichen homologen 1-Phenylcumarone, die bisher noch nicht bekannt waren, sicherzustellen, haben wir ihre Synthese aus homologen Salicylaldehyden und Phenyl-chlor-essigsäure nach dem Verfahren von Stoermer und Reuter ${ }^{2}$ ) in Angriff genommen:

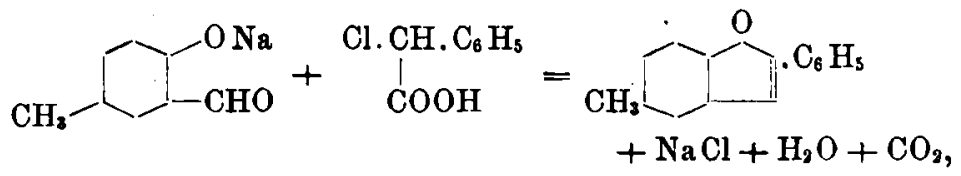

wobei sich eine völlige Identität des synthetischen mit dem durch Phenylwanderung entstandenen Produkt ergab.

Für die 1-Phenylcumarone charakteristisch ist die Gelblärbuug mit konzentrierter Schwefelsäure, die bald in Grün umschlägt.

Die durch direkte Bromierung der 2-Phenylcumarone entstehenden 1-Brom-2-phenyl-cumarone zeigen in mancher Richtung eine ziemlich auffallende Beweglichkeit des Bromatoms. So lassen sie sich bei der Einwirkung salpetriger Dämpfe ziemlich glatt in 1-Nitro-2phenyl-cumarone überführen, gerade wie das 1-Bromcumaron selbst,

1) K. Hildobrandt, Zur Kenntnis phenylierter Cumarone, Dissertation, Rostock 1906.

2) B. 36, 3979 [1903].

Berichte d. D. Chem. Gesellschaft. Jahrg. XXXXIV. 
das nach Stoermer und Kahlert') so sehr gut in 1-Nitrocumaron übergeht. Sehr viel einfacher gestaltet sich das Verfahren, wenn man das gebromte Cumaron in Eisessig löst, einige Kubikzentimeter konzentrierter Salpetersüure und dann etwas Natriumnitrit binzugibt und nun so lange erwärmt, bis keine Bromdämpfe mehr entweichen. Man erhält so den Nitrokörper beim Eingießen in Wasser sogleich fast rein. Nach den Untersuchungen besonders von Wallach ${ }^{2}$ ) und von Wieland ${ }^{3}$ ) kann es wohl keinem Zweifel mehr unterliegen, daß man es hierbei ebenfalls mit einer Anlagerung von Stickstofftrioxyd und einer dann nachiolgenden Abspaltung von Nitrosylbromid zu tun hat:

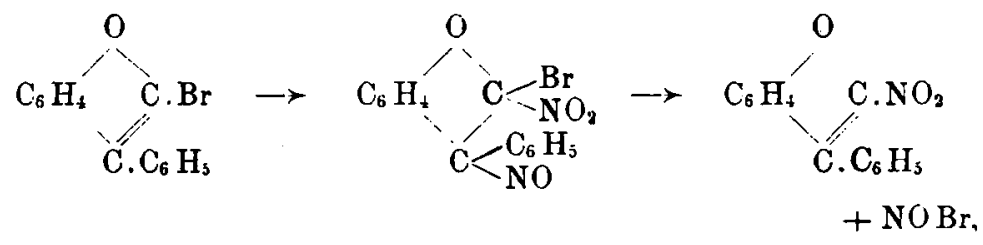

wenngleich Anlagerungsprodukte nicht gefaßt werden können. Die isomeren 1-Phenyl-2-brom-cumarone setzen sich mit salpetriger Säure in diesem Sinne nicht um.

Bei der Einwirkung von Phosphoroxychlorid unter Druck auf die oben angeführten Lactone entstehen sehr glatt die entsprechendeu 1-Chlor-2-phenyl-cumarone, ohne da $\beta$ dabei jemals, wie auch zu erwarten, eine Phenylwanderung $z u$ beobachten gewesen wäre. Diese so leicht zugänglichen Körper lassen sich nicht zu den chlorfreien Verbindungen reduzieren, weder durch Phosphortribromid, noch durch andere Reduktionsmittel. Natrium und Alkohol führt sogleich in die entsprechenden Phenylcumarane über, die bei der Einwirkung von Brom das Halogen nicht mehr im Furanring, sondern in Benzolkern fixieren. Derartige im Benzolkern balogenierte Phenylcumarane lassen sich nicht darstellen durch Reduktion der entsprechenden Cumarone oder 1-Chlorcumarone, denn in allen Fällen entsteht das nichtsubstituierte 2-Phenylcumaran, indem auch das Halogen des Benzolkerns durch die Einwirkung von Natrium und Alkohol herausgenommen wird.

Endlich sei noch erwähnt, daß alle Versuche, 1-Phenyl-cumarone, entsprechend der bekannten Anschützschen Synthese von Stilben aus Zimtsäurephenylester, durch trockne Destillation von Cumarilsäurephenylestern zu erhalten, völlig fehlschlugen.
1) B. 35, 1638 [1902].
2) A. 382, 305 [1904].
3) A. $329,225[1903]$. 
Experimentelles.

(Nach Versuchen des Hrn. Claus Decker.)

2-Phenyl-und 1-Phenyl-4-methyl-cumaron,

1.<smiles>CCCCCCCCCCCCCCCCCCCCCCC</smiles>

2.

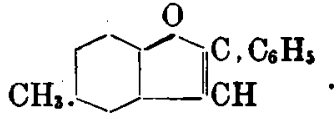

Das nach den Angaben von Bistrzycki und Simonis') leicht in guter Ausbeute gewinnbare Phenyl-p-kresylessigsäurelacton läßt sich sowohl durch Erbitzen mit Phosphortribromid wie mit Phosphorpentasulfid in 2-Phenyl-4-methylcumaron überführen.

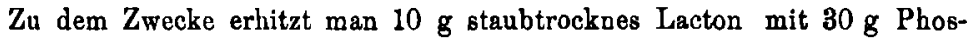
phortribromid in einem langbalsigen, mit angeschmolzenem Steigrohr versehenen Kolben 14 Stunden auf $190^{\circ}$ im Ölbade, wobei nach anfanglichem Entweichen von Bromwasserstoff der Kolbeninhalt eine rote Farbe und ziemlich dickflüssige Konsistenz bekommt. Gießt man dann in Wasser (Phosphorwasserstoff-Entwicklung!) und leitet in die stark alkalisch gemachte Lösung einen Wasserdampistrom, so geht das 2-Phenyl-4-methyl-cumaron als farbloses $O \mathrm{l}$ über, das nach dem Ausăthern destilliert wird. Ausbente $1.5 \mathrm{~g}$.

In ãhnlicher Weise entsteht derselbe Körper bei viertelstūndigem $\mathrm{Er}$ bitzen einer innigen Mischung von $5 \mathrm{~g}$ Lacton mit $12 \mathrm{~g}$ Phosphorpentasulfid in Ölbade auf 200-210 . Das Präparat ist aber gelblich gefärbt und besitzt einen höchst unangenebmen Geruch, der sich nur bei lăngerem Erwărmen auf dem Wasserbade einigermaßen verliert. Ausbeute $0.6 \mathrm{~g}$

Das reine 2-Phenylmethyleumaron siedet bei $193^{\circ}$ bei $20 \mathrm{~mm}$ Druck, erstarrt beim Abkühlen nicht und färbt sich wie alle 2-Phenylcumarone mit konzentrierter Schwefelsäure intensiv rot. Geruch süßlich aromatisch.

$0.1032 \mathrm{~g}$ Sbst.: $0.3283 \mathrm{~g} \mathrm{CO}_{2}, 0.554 \mathrm{~g} \mathrm{H}_{2} \mathrm{O}$.

$$
\begin{aligned}
& \mathrm{C}_{15} \mathrm{H}_{12} \mathrm{O} \text {. Ber. C 86.54, H } 5.77 . \\
& \text { Gef. } 86.76 \text {, } 6.00 .
\end{aligned}
$$

- Erbitzt man $3 \mathrm{~g}$ 2-Phenylmethylcumaron mit $50-60 \mathrm{~g}$ absolutem Alkohol und $10 \mathrm{~g}$ Natrium, so erbält man bei nachfolgender Wasserdampt-Destillation leicht das alsbald krystallinisch. erstarrende 2-Phenyl-4-methyl-cumaran in einer Ausbeute von $90 \%$, das weiße Prismen vom Schmp. 57 $7^{\circ}$ bildet und sich als identisch mit dem schon früher von Stoermer und Kippe') dargestellten Produkt erwies.

Bleibt man bei der Einwirkung von Tribromid auf das Lacton im zugeschmolzenen Rohr unter der Temperatur von $230^{\circ}$, so entsteht auch fast nur die 2-Phenylverbindung, während bei $255^{\circ}$ schon fast völlige Verkohlung eintritt. Erhitzt man das Lacton $(7.5 \mathrm{~g})$ dagegen
1) B. 31, 2812 [1898].
2) B. 36, 4001 [1903]. 
mit $25 \mathrm{~g}$ Tribromid vorsichtig auf $235-238^{\circ}$ und erhält die Mischung bei dieser Temperatur 18-20 Stunden, so ist der Robrinhalt dickflüssig und dunkelgrün gefärbt, und es entweicht beim Öffnen eine große Menge von Bromwasserstoff. Bei der Dampfdestillation geht ein Öl über, das alsbald teilweise zu Krystallen erstarrt, die, auf Ton abgepreßt und aus verdünntem Alkohol umkrystallisiert, den Schmp. $129^{\circ}$ zeigen.

Dieses 1-Phenyl-4-methyl-cumaron bildet rein weiße, verfilzte Nädelchen, die mit konzentrierter Schwefelsäure sich zuerst citronengelb und nach kurzer Zeit grün färben, eine Färbung, die auch bei längerem Steben kaum verblaßt ${ }^{1}$ ).

Zur sicheren Feststellung der Konstitution der Substanz wurden $6 \mathrm{~g}$ Homosalicylaldehyd ( $\mathrm{CHO}: \mathrm{OH}: \mathrm{CH}_{3}=3: 4: 1$ ) (1.5 Mol.), $9 \mathrm{~g}$ Phenylchloressigsäure (1.5 Mol.) und $4 \mathrm{~g}$ Natriumhydroxyd (3 Mol.) mit $14 \mathrm{~g}$ Alkohol und $8 \mathrm{~g}$ Wasser im Rohr 15-18 Stunden auf 205-210 erhitzt und der Rohrinhalt (starker Druck!) danach mit Wasserdampf destilliert"). Das übergehende 1-Phenyl-4-methylcumaron erstarrt schon im Kühler und schmilzt bei $129^{\circ}$, eine Mischprobe mit dem obigen bei $128.5-129^{\circ}$. Ausbeute gering.

$$
\begin{aligned}
0.0982 \mathrm{~g} \text { Sbst.: } 0.312 \mathrm{~g} \mathrm{CO}_{2}, 0.0521 \mathrm{~g} \mathrm{H}_{2} \mathrm{O} . \\
\mathrm{C}_{15} \mathrm{H}_{12} \mathrm{O} \text {. Ber. C } 86.54 \text {, H } 5.77 . \\
\text { Gef. 》 } 86.68 \text {, } 5.96 .
\end{aligned}
$$

Brom wird von 2-Phenyl-4-methylcumaron in Schwefelkohlenstofflösung zumal im Sonnenlicht unter reichlicher BromwasserstoffEntwicklung sofort aufgenommen. Nach dem Abdunsten des Lösungsmittels krystallisieren sofort sternförmig gruppierte Nadeln von 1-Brom-2-phenyl-4-methyl-cumaron aus, die, aus Alkohol umkrystallisiert, bei $65^{\circ}$ schmelzen und mit konzentrierter Schwefelsäure nur eine schwache Rotfärbung zeigen.

$0.1451 \mathrm{~g}$ Sbst.: $0.0920 \mathrm{~g} \mathrm{AgBr}$.

$$
\mathrm{C}_{15} \mathrm{H}_{11} \text { Br O. Ber. Br 26.94. Gef. Br 26.98. }
$$

Alle Versuche, denselben Körper aus dem Phenyl-p-kresyl-bromessigsäurelacton vom Schmp. $96^{\circ}$ durch Reduktion und Umlagerung

1) Bei Gelegenheit der Darstellung obiger Verbindung wurde durch besondere Versuche festgestellt, dab die 2-Phenglverbindung, und $z$ war das leichter zugăngliche 2-Phen yl-cu maron selbst sich nicht durch längeres Erhitzen mit Tribromid auf $235^{\circ}$ sekundär in 1-Phenylcumaron umlagert. Beim Öffnen des Robres zeigte sich gar kein Druck, und das leicht identifizierbare 2-Phenylcumaron (Stoermer und Kippe, B. 36, 4005 [1903]) warde quantitativ wiedergewonnen. Ebenso verhielt sich das durch direkte Bromierung gewonnene 1-Brom-2-phenyl-cumaron, das bei gleicher Behandlung ebenfalls fast quantitativ zurückgewonnen wurde und noch den gleichen Bromgehalt besaß. (Ber. $29.3 \%$. Gef. $29.6 \%$.)

2) Vergl. B. 36, 3979 [1903]. 
unter dem Einfluß ron Phosphortribromid oder -pentasulfid zu erhalten, hatten nur ein negatives Ergebnis ${ }^{1}$ ).

Läßt man auf die Bromverbindung unter einer Glasglocke die Dämple von salpetriger Säure einwirken, so entsteht langsam das 1-Nitro-2-phenyl-4-metbyl-cumaron, das man bequemer erhält, wenn man $2 \mathrm{~g}$ Bromid in $10 \mathrm{ccm}$ Eisessig löst und nach Züsatz von etwas konzentrierter Salpetersäure und Natriumnitrit erwärmt, bis kein Brom mebr entweicht. GieBc man dann in Wasser, so scheiden sich aus der Flüssigkeit gelbe Flocken aus, die, nach dem Auswaschen auf Ton getrocknet und wiederholt aus Alkohol umkrystallisiert, kleine, strahlig gruppierte, gelbe Nadeln vom Scbmp. $115-116^{\circ}$ bilden. Nicht flüchtig mit Wasserdämpfen.

$0.1074 \mathrm{~g}$ Sbst.: $0.2800 \mathrm{~g} \mathrm{CO}_{2}, 0.0422 \mathrm{~g} \mathrm{H} \mathrm{H}_{9} \mathrm{O},-0.2810 \mathrm{~g}$ Sbst.: $13.5 \mathrm{ccm}$ $\mathrm{N}\left(13^{\circ}, 769 \mathrm{~mm}\right)$.

$$
\begin{array}{ll}
\mathrm{C}_{15} \mathrm{H}_{11} \mathrm{O}_{3} \mathrm{~N} . & \text { Ber. } \mathrm{C} 71.15, \mathrm{H} 4.34, \mathrm{~N} 5.53 . \\
& \text { Gef. } ₫ 71.02, \star 4.69, \star 5.77 .
\end{array}
$$

Das Bromid reagiert auch leicht mit Piperidin unter Abscheidung von bromwasserstoffsaurem Piperidin, doch gelang es nicht, die entsprechende Piperidoverbindung $\mathrm{zu}$ isolieren ${ }^{2}$ ).

1) Dasselbe war der Fall mit dem Oxydiphenylbromessigsäarelacton, das gewonnene bromhaltige $O 1$ besaß nur einen Bromgehalt von $8 \%$ statt $29.3 \%$.

3) Bei Gelegenheit dieser Versuche wurde auch die Bromierung des 2- und 1-Phenylcumarons vorgenommen und das Verhalten der Bromide gegen salpetrige Säure geprüft. Die Bromierung. erfolgt leicht, wie oben, in Schwefelkohlenstoff. Das 1-Brom-2-phengl-cumaron ist ein süblich riechendes Öl vom Sdp. $195^{\circ}$ bei $20 \mathrm{~mm}$ Druck, das auf keine Weise erstarrt.

\subsection{6 g Sbst.: $0.1340 \mathrm{~g} \mathrm{AgBr}$.}

$\mathrm{C}_{14} \mathrm{H}_{9} \mathrm{OBr}$. Ber. Br 29.3. Gef. Br 29.45.

Durch Reduktion mit Natrium und Alkohol entsteht das bekannte 2-Phenglcumaran vom Schmp. 38-39 . Bei der Behandlung mit konzentrierter Salpetersäure und Natriumnitrit in Eisessiglösung (wie oben) erhălt man leicht das bei $105^{\circ}$ schmelzende, in schönen, langen, gelben Nadeln krystallisierende 1-Nitro-2-phenyl-cu maron, leicht löslich in heißem Alkohol, 6chwerer in Ligroin. Durch Reduktion ist eine Amidoverbindung nicht zu erhalten.

$0.1910 \mathrm{~g}$ Sbst.: $0.5300 \mathrm{~g} \mathrm{CO}, 0.0748 \mathrm{~g} \mathrm{H}_{2} \mathrm{O} .-0.2110 \mathrm{~g}$ Sbst.: $10.1 \mathrm{ccm}$ $\mathrm{N}\left(12^{\circ}, 761 \mathrm{~mm}\right)$.

$$
\begin{aligned}
& \mathrm{C}_{14} \mathrm{H}_{9} \mathrm{O}_{3} \mathrm{~N} \text {. Ber. C 75.35, H 4.0, N 5.84. } \\
& \text { Gef. \75.68, } 4.43 \text {, \5.74. }
\end{aligned}
$$

1-Phenylcumaron, erhalten in einer Ausbeute von $36 \%$ beim Erhitzen von $5 \mathrm{~g}$ Oxydiphenylessigsăurelacton mit $17.5 \mathrm{~g}$ Phosphortribromid wăhrend 18-20 Stunden auf $235^{\circ}$ im Rohr, läBt sich ebenso wie das Isomere leicht 
2-Phenyl-und 1-Phenyl-5-methyl-cumaron,

3.<smiles>CCCCCCCCCCCCCCCCCCCC</smiles>

4.

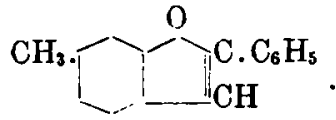

Die Konstitution des Ausgangsmaterials, des Phenyl-m-kresylessigsäurelactons, ist von den Entdeckern, Bistrzycki und F latau ${ }^{2}$, offen gelassen worden. Es wurde daher zunächst besonderer Wert auf die Einheitlichkeit des Lactons gelegt, und als sich diese berausstellte, die Konstitution auf Grund folgender Überlegung ermittelt. Die Synthese aus Mandelsäure und $m$-Kresol läßt die Wahl zwischen den Formeln 5 und 6:

5 .

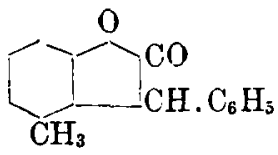

6.<smiles>CCCCCCCCCCCCC1OC(=O)C2CCCCC12</smiles>

Führt man $m$-Kresol in den zugehörigen Salicylaldebyd über, dessen Konstitution bekannt ist $\left(\mathrm{CH}_{3}: \mathrm{OH}: \mathrm{CHO}=1: 3: 4\right)$, so läßt sich aus diesem durch Kondensation mit Phenyl-chloressigsäure dasselbe 1-Phenyl-5-methylcumaron (4) gewinnen, das bei Phenylwanderung aus dem Lacton durch Umsetzung mit Phosphortribromid zu erwarten war. Da diese sich als identisch erwiesen, so muß dem Lacton die Formel 6 und nicht die isomere 5 zukommen.

Erhitzt man das Phenyl-m-kresylessigsäurelacton (15 g) im Rohr vorsichtig mit Phosphoroxychlorid (10 g) 15 Stunden lang auf $130^{\circ}$ ), so entsteht mit wechselnder Ausbeute (im günstigsten Falle $60 \%$ ) das 1-Chlor-2-phenyl-5-methyl-cumaron.

Der Rohrinhalt ist dickflüssig und dunkelgrün. beim Öffnen ist starker Druck vorhanden. Man löst in Wasser, digeriert mit Natronlauge und destilliert den getrockneten Ätherauszag, wobei das Chlorid bei $10 \mathrm{~mm}$ Druck und $175^{\circ}$ als bald erstarrender Körper übergeht, der aus verdfinntem Alkohol in silberglänzenden Nadeln vom Schmp. $85.5^{\circ} \mathrm{krystallisiert.}$

$0.1590 \mathrm{~g}$ Sbst.: $0.0964 \mathrm{~g} \mathrm{Ag} \mathrm{Cl}$.

$$
\mathrm{C}_{15} \mathrm{H}_{11} \mathrm{OCl} \text {. Ber. Cl 14.46. Gef. Cl } 14.90 \text {. }
$$

bromieren. Das Bromid siedet bei $203^{\circ}$ und $23 \mathrm{~mm}$ Druck, erstarrt alsbald und krystallisiert aus verdunntem Alkohol in silberglänzenden Blāttchen vom Schmp. 810. Konzentricrte Schwefelsäure gibt nur schwache Rotfärbung, salpetrige Säure ist ohne Einwirkung.

0.1452 g Sbst.: $0.1010 \mathrm{~g} \mathrm{AgBr}$.

$\mathrm{C}_{14} \mathrm{H}_{9} \mathrm{OBr}$. Ber. Br 29.3. Gef. Br 29.6 .

1) B. 30, 130 [1897].

) Bei $120^{\circ}$ ist meist keine Einwirkung zu beobachten, oberhalb $130^{\circ}$ häufig völlige Verkohlung. 
Reduziert man die Chlorverbindung (oder das Bromid, s. u.) mit Natrium und Aikobol (vergl. oben), so entsteht leicht und glatt das bei $45^{\circ}$ scbmelzende, in kleinen weißen Nadeln krystallisierende 2-P hen yl5 -methyl-cumaran. Sdp. $170^{\circ}$ bei $14 \mathrm{~mm}$ Druck.

$0.1398 \mathrm{~g}$ Sbst.: $0.4192 \mathrm{~g} \mathrm{CO}_{2}, 0.0791 \mathrm{~g} \mathrm{H}_{2} \mathrm{O}$.

$$
\begin{aligned}
& \mathrm{C}_{15} \mathrm{H}_{14} \text { O. Ber. C 85.71, H } 6.66 . \\
& \text { Gef. \$ 86.04, } 6.73 .
\end{aligned}
$$

Bei der Behandlung dieses Cumarans (2.5 g) in Eisessig (25 ccm) mit Brom (2 $\mathrm{g}$ in $10 \mathrm{ccm}$ Eisessig) erbält man leicht das in derben, spießigen Nadeln krystallisierende 4(?)-Brom-2-phenyl-5-methylcumaran vom Schmp. $125^{\circ}$, aus dem durch Alkali kein Halogen mehr abspaltbar ist.

$0.2361 \mathrm{~g}$ Sbst.: $0.1541 \mathrm{~g} \mathrm{AgBr}$.

$\mathrm{C}_{13} \mathrm{H}_{13} \mathrm{O}$ Br. Ber. $\mathrm{Br}$ 27.68. Gef. $\mathrm{Br} 27.87$.

Erbitzt man das 2-Phenyl-5-methylcumaran ( $3 \mathrm{~g}$ ) mit $7 \mathrm{~g}$ Ätzkali und $50 \mathrm{ccm}$ Alkobol 18 Stunden im Autoklaven auf $200^{\circ}$, so findet völlige Aufspaltung des Furanringes statt, und es entsteht in einer Ausbeute von $80 \%$ das Phenyl-m-kresyl-äthylen,

$$
\mathrm{C}_{6} \mathrm{H}_{3}\left(\mathrm{CH}_{3}\right)<\mathrm{CH}\left(\mathrm{C}_{6} \mathrm{H}_{3}\right): \mathrm{CH}_{2},
$$

ein fast farbloses, nicht erstarrendes, alkalilösliches Öl, das im Gegensatz zu dem Cumaran sich mit konzentrierter Schwefelsäure intensiv rot färbt. Es läßt sich, wie analoge Verbindungen '), auch aus alkalischer Lösung ausäthern. Sdp. $205^{\circ}$ bei $20 \mathrm{~mm}$ Druck.

0.1312 g Sbst.: $0.4100 \mathrm{~g} \mathrm{CO}_{2}, 0.0812 \mathrm{~g} \mathrm{H}_{2} \mathrm{O}$.

$$
\begin{aligned}
& \mathrm{C}_{15} \mathrm{H}_{14} \mathrm{O} \text {. Ber. C 85.71, H 6.66. } \\
& \text { Gef. - 85.23, > 6.90. }
\end{aligned}
$$

Die Darstellung des 2-Phenyl-5-methyl-cumarons aus dem Lacton der Phenyl- $m$-kresylessigsäure mittels Phosphortribromids oder Phosphorpentasulfids gestaltet sich gerade so wie oben für das Isomere angegeben. Die Ausbeute bei der Darstellung mit Tribromid beträgt $2.3 \mathrm{~g}$ aus $15 \mathrm{~g}$ Lacton, mit Pentasulfid ist sie kaum halb so grob. Die Verbindung bildet derbe Nadeln vom Schmp. $31^{\circ}$ und von schwachem, süßlichem Geruch. Sdp. $168^{\circ}$ bei $18 \mathrm{~mm}$ Druck. Die intensiv orangerote Färbung mit konzentrierter Schwefelsäure verblaßt rasch beim Erwärmen.

$0.1086 \mathrm{~g}$ Sbst.: $0.344 \mathrm{~g} \mathrm{CO}_{2}, 0.0602 \mathrm{~g} \mathrm{H}_{2} \mathrm{O}$.

$$
\begin{aligned}
& \mathrm{C}_{15} \mathrm{H}_{12} \mathrm{O} \text {. Ber. C 86.54, H 5.76. } \\
& \text { Gef. * } 86.39, » 6.20 \text {. }
\end{aligned}
$$

1) Stoermer und Kippe, B. 36, 3998 [1903]. 
Beim Bromieren des Cumarons in Schwefelkohlenstofflösung im Sonnenlicht erbält man leicht das bei $95^{\circ}$ schmelzende 1-Brom-2phenyl-5-methyl-cumarou, das sich mit konzentrierter Schwefelsäure vur vorübergehend blaßrot färbt.

$0.2230 \mathrm{~g}$ Sbst.: $0.1420 \mathrm{~g} \mathrm{AgBr}$.

$\mathrm{C}_{15} \mathrm{H}_{11} \mathrm{O}$ Br. Ber. Br 26.94. Gel. Br 27.1.

Durch Einwirkung von Salpetersäure und Nitrit in Eisessiglösung geht es leicht in das bei $119-120^{\circ}$ schmelzende 1-Nitro-2phenyl-5-methyl-cumaron über, das in kleinen, gelben Krystallwarzen aus Alkohol krystallisiert.

$0.1075 \mathrm{~g}$ Sbst.: $0.2822 \mathrm{~g} \mathrm{CO}_{2}, 0.0432 \mathrm{~g} \mathrm{H}_{2} \mathrm{O} .-0.2087 \mathrm{~g}$ Sbst.: $9.7 \mathrm{ccm}$ $\mathrm{N}\left(8^{\circ}, 772 \mathrm{~mm}\right)$.

$\mathrm{C}_{15} \mathrm{H}_{11} \mathrm{O}_{3} \mathrm{~N}$. Ber. C 71.15, H 4.34, N 5.53.

Gef. \71.59, $459, \gg 5.80$.

Die Umwandlung des Lactons in das 1-Phenyl-5-methylcumaron erfolgt hier ebenfalls nur schwierig und mit geringer Ausbeute. Die Gewinnung ist genau die gleiche wie bei dem obigen Isomeren, nur ist die Isolierung schwieriger, weil das bei der Wasserdampf-Destillation auskrystallisierende Produkt, das unscharf $z$ wischen $100^{\circ}$ und $102^{\circ}$ schmilzt, ein Gemisch des bochschmelzenden Isomeren mit dem rom Schmp. $31^{\circ}$ ist. Beim Auswaschen mit Alkobol und mehrmaligem Umkrystallisieren stieg der Schmelzpunkt bis auf $135.5^{\circ}$, wo er konstant blieb. Die erbaltenen kleinen, silberglänzenden Nädelchen gaben mit konzentrierter Schwefelsäure jetzt eine intensiv gelbe Färbung, die allmählich in Dunkelgrūn überging. Auch bier konnte die Identität mit dem durch Kondensation erbaltenen 1-Phenyl-5methylcumaron leicht durch Vergleich und Mischprobe erwiesen werden. Aber auch die Ausbeute an diesem Produkt, gewonnen (wie oben) aus reinem $m$-Homosalicylaidehyd vom Schmp. 6101), Phenylchloressigsäure und Alkali im Rohr bei $200-205^{\circ}$, läßt sehr zu wünschen übrig. Der Schmelzpunkt der Mischprobe lag genau bei $135^{\circ}$.

$0.1190 \mathrm{~g}$ Sbst.: $0.3780 \mathrm{~g} \mathrm{CO}_{2}, 0.0659 \mathrm{~g} \mathrm{H}_{2} \mathrm{O}$.

$\mathrm{C}_{15} \mathrm{H}_{12} \mathrm{O}$. Ber. C 86.54, H 5.76 .

Gef. » $86.69, \rtimes 6.10$.

2-Anisyl-cumaron und 2-Anisyl-4-methyl-cumaron.

Zur Gewinnung des 2-Anisylcumarons bedurfte es des bisher nicht bekannten $0.0 x y$ phenyl-anisylessigsäurelactons, das in ähnlicher Weise wie die anderen Lactone aus Anisaldehyd-cyanbydrin, Phenol und 73-prozentiger Schwefelsäure gewonnen wurde.

1) Vergl. Fries und Klostermann, B. 39, 872 Anm. 2 [1906]. 
$10 \mathrm{~g}$ Cyanhydrin, mit $12 \mathrm{~g}$ Phenol innig gemischt, werden langsam in $50 \mathrm{~g}$ 73-prozentiger Schwefelsäure eingetragen. Die bald lebhaft einsetzende Reaktion ist nach ca. $1 / 4$ Stunde beendigt, wonach der feste Rückstand mit Sodalösung digeriert wird. Das schwach gelb bis rot gefärbte Pulver wird aus verdünntem Alkohol umkrystallisiert, bis die farblosen Nadeln bei $178^{\circ}$ scbmelzen.

0.2481 g Sbst.: $0.6800 \mathrm{~g} \mathrm{CO}_{2}, 0.1180 \mathrm{~g} \mathrm{H}_{2} \mathrm{O}$.

$$
\begin{aligned}
& \mathrm{C}_{15} \mathrm{H}_{12} \mathrm{O}_{3} \text {. Ber. C 75.00, H 5.00. } \\
& \text { Gef. 74.90, 5.32. }
\end{aligned}
$$

Leider gelang es nicht, bei der Einwirkung von Tribromid oder Pentasulfid das zugehörige Anisylcumaron zu erhalten; nur Spuren eines festen Körpers waren faßbar, die im Gegensatz zu dem Lacton sich mit konzentrierter Schwefelsäure intensiv rot färbten. Dagegen war es möglich, aus dem homologen $p$-Kresyl-anisyl-essigsäurelacton das betreffende Anisyl-methyl-cumaron ${ }^{1}$ ) darzustellen.

Die Darstellung des Lactons erfolgte wie oben aus $7 \mathrm{~g}$ Anisaldehydeyanhydrin, $10 \mathrm{~g} p$-Kresol und $40 \mathrm{~g}$ 73-prozentiger Schwefelsāure. Es bilket schöne, weiße Blättchen rom Schmp. 135\%. Ausbeute $60 \%$.

$0.1680 \mathrm{~g}$ Sbst.: $0.4637 \mathrm{~g} \mathrm{CO}_{2}, 00885 \mathrm{~g} \mathrm{H}_{2} \mathrm{O}$.

$$
\begin{array}{ll}
\mathrm{C}_{16} \mathrm{H}_{14} \mathrm{O}_{3} . & \text { Ber. C 75.59, H 5.51. } \\
& \text { Gef. } \$ 75.27, \star 5.86 .
\end{array}
$$

Erhitzt man $10 \mathrm{~g}$ des Lactons mit $25 \mathrm{~g}$ Tribromid 12 Stunden auf $220^{\circ}$ und behandelt das Reaktionsprodukt in alkaliscber Lösung mit Wasserdampf, so geht das alsbald krystallinisch erstarrende 2-Anisyl-4-methyl-cumaron über, das aus verdünntem Alkohol in verfilzten Nadeln vom Schmp. $73.5^{\circ}$ herauskommt und mit konzentrierter Schwefelsäure eine prachtiolle Eosinfärbung gibt. Ausbeute nur $4 \%$.

0.1622 g Sbst.: $0.4789 \mathrm{~g} \mathrm{CO}_{2}, 0.0890 \mathrm{~g} \mathrm{H}_{2} \mathrm{O}$.

$\mathrm{C}_{16} \mathrm{H}_{14} \mathrm{O}_{2}$. Ber. C 80.67, $\mathrm{H}$ 5.88.

Gef. \80.51, 》 6.04 .

Phosphorpentasulfid gibt noch erheblich schlechtere Ausbeute. Eine Wanderung des Anisylrestes iu die 1-Stellung konnte hier, des geringen Beobachtungsmaterials wegen, nicht festgestellt werden. Bei der Reduktion mit Natrium und Alkohol erhält man ein (nicht genauer untersuchtes) Öl, wohl das zugehörige Cumaran.

Einwirkung von Phosphoroxychlorid und -tribromid auf halogenierte Lactone.

(Bearbeitet von K. Hildebrandt.)

Die für die Untersucbung notwendigen Lactone wurden nach der allgemeinen Methede aus $p$-Chlorphenol bezw. $p$-Bromphenol und Mandelsäure

1) Dargestellt von KarI Hildebrandt. Nissert., Rostock 1906, S. 43. 
gewonnen. Ersteres (l) bildet schöne, weißo Nadeln rom Schmp. $125^{\circ}$, ist leicht löslich in Benzol und Chloroform, sowie in heiBem Alkohol und Bisessig, letzteres (II) stellt eben solche Nädelchen vom Schmp. $123^{\circ}$ dar. Die Ausbenten betragen in beiden Fällen ca. $60 \%$ der Theorie.

I. $0.2153 \mathrm{~g}$ Sbst.: $0.5412 \mathrm{~g} \mathrm{CO}_{2}, 0.0724 \mathrm{~g} \mathrm{H}_{2} \mathrm{O} .-0.1890 \mathrm{~g}$ Sbst.: $0.1104 \mathrm{~g}$ Ag Cl.

$$
\begin{aligned}
& \mathrm{C}_{14} \mathrm{H}_{9} \mathrm{O}_{2} \mathrm{Cl} \text {. Ber. C 68.71, H 3.68, Cl } 14.51 . \\
& \text { Gef. » } 68.55 \text {, } 3.71 \text {, } 14.44 .
\end{aligned}
$$

II. $0.2187 \mathrm{~g}$ Sbst.: $0.4652 \mathrm{~g} \mathrm{CO}_{2}, 0.0648 \mathrm{~g} \mathrm{H}_{2} \mathrm{O} .-0.2318 \mathrm{~g}$ Sbst.: $0.1514 \mathrm{~g}$ $\mathrm{AgBr}$.

$$
\begin{aligned}
& \mathrm{C}_{14} \mathrm{H}_{9} \mathrm{O}_{2} \mathrm{Br} \text {. Ber. C 58.12, H 3.14, Br 27.66. } \\
& \text { Gef. } 57.98 \text {, } 3.29, \text { > } 27.78 .
\end{aligned}
$$

Erhitzt man $0-0 x y-p \cdot$ chlordiphenyl-essigsäurelacton $(15 \mathrm{~g}) \mathrm{mit}$ Phosphoroxychlorid $(9.5 \mathrm{~g})$ im Rohr langsam auf $145^{\circ}$ und erhält den Inhalt dann noch 9 Stunden auf dieser Temperatur, so bildet der Rohrinhalt eine bräunliche, dicke, mit Nadeln durchsetzte Flüssigkelt. Diese wird in Wasser gegossen, mit Natronlauge stark alkaliscb gemacht, erbitzt und dann mit Äther extrahiert. Der Ätherrückstand, das 1-Chlor-2-phenyl-4'-chlor-cumaron, krystallsiert aus Alkohol in schönen, langen, silberglänzenden Nadeln vom Schmp. $122^{\circ}$. Ziemlich leicht löslich in allen organischen Lösungsmittelu. Ausbeute ca. $60 \%$ der Theorie. Ag Cl.

$01297 \mathrm{~g}$ Sbst.: $0.3032 \mathrm{~g} \mathrm{CO}_{2}, 0.0378 \mathrm{~g} \mathrm{H}_{2} \mathrm{O} .-0.1302 \mathrm{~g}$ Sbst.: $0.1430 \mathrm{~g}$

$$
\begin{aligned}
& \mathrm{C}_{14} \mathrm{H}_{8} \mathrm{O} \mathrm{Cl}_{2} \text {. Ber. C 63.87, H 3.04, Cl 26.96. } \\
& \text { Gef. » 63.76, 》 3.23, » } 27.21 \text {. }
\end{aligned}
$$

Durch Behandeln mit Alkohol und Natrium entsteht lediglich das schon bekannte 2-Phenylcumaran vom Scbmp. $39^{\circ}$.

Erhitzt man das Lacton (10 g) mit Phosphortribromid (25 g) am Steigrohr 12 Stunden im Ölbade auf $200^{\circ}$, so entsteht in einer Ausbeute von $30 \%$ das 2-Phenyl-4'-chlor-cumaron vom Schmp. $34^{\circ}$ (Isolierung wie oben). Es ist leicht löslich in den gewöhnlichen organischen Lösungsmitteln und färbt sich mit konzentrierter Schwefelsäure leucbtend orange. Die gleiche Verbindung entstebt auch mit Phosphorpentasulfid in einer Ausbeute von $20 \%$.

$0.1502 \mathrm{~g}$ Sbst.: $0.4041 \mathrm{~g} \mathrm{CO}_{2}, 0.0558 \mathrm{~g} \mathrm{H}_{2} \mathrm{O} .-0.2083 \mathrm{~g}$ Sbst.: $0.1350 \mathrm{~g}$ $\mathrm{AgCl}, 0.2973 \mathrm{~g}$ Sbst.: $0.1901 \mathrm{~g} \mathrm{AgCl}$.

$$
\begin{aligned}
& \mathrm{C}_{14} \mathrm{H}_{9} \mathrm{OCl} \text {. Ber. C 73.52, H 3.94, Cl 15.53. } \\
& \text { Gef. } \$ 73.36, \gg 4.12 \text {, » 15.68, } 15.79 .
\end{aligned}
$$

Zahlreiche Versuche, mit Hilfe von Tribromid im Rohr bei böherer Temperatur das 1-Phenyl-4'-chlor-cumaron zu gewinnen, 
schlugen fehl; es wurde niemals ein höher schmelzendes Produkt beobachtet.

Die Darstellung des 1-Chlor-2-phenyl-t'-brom-cumaronserfolgte genau, wie angegeben, aus dem bromierten Lacton. Der Körper bildet lange, glänzende Nadeln rom Schmp. $119^{\circ}$ aus verdïnntem Alkohol. Ansbeute ca. $60 \%$.

$02861 \mathrm{~g}$ Sbst.: $0,3089 \mathrm{~g} \mathrm{AgCl}+\mathrm{AgBr}=0.1355 \mathrm{~g} \mathrm{AgCl}, 0.1734 \mathrm{~g}$ $\operatorname{AgBr}$.

$$
\begin{aligned}
& \mathrm{C}_{14} \mathrm{H}_{\mathrm{B}} \mathrm{OClBr} \text {. Ber. Cl 11.52, Br 26.01. } \\
& \text { Gef. } 11.70, \text { 25.76. }
\end{aligned}
$$

Das mit Hilfe von Phosphortribromid oder -pentasulfid gewonnene 2.Phenyl-4'-brom-cumaron bildet, ans verdünntem Alkohol krystallisiert, lange, verfilzte Nadeln rom Schmp. $38^{\circ}$, die sich mit konzentrierter Schwefelsäure prachtvoll orangerot färben. Ausbeute ca. $20 \%$ der Theorie.

0.2120 g Sbst.: $0.4775 \mathrm{~g} \mathrm{CO}_{2}, 0.0646 \mathrm{~g} \mathrm{H}_{2} \mathrm{O} .-0.1625 \mathrm{~g}$ Sbst.: $0.1109 \mathrm{~g}$ $\mathrm{AgBr}$.

$\mathrm{C}_{14} \mathrm{H}_{9} \mathrm{OBr}$. Ber. C $61.54, \mathrm{H} 3.29, \mathrm{Br} 29.28$.
Gef. 61.41 , 3.34, 29.04.

Auch hier war es nicht möglich, die durch Phenylwanderung entstehende 1-Pbenylverbindung $\mathrm{zu}$ isolieren.

Rostock, im Juni 1911.

\section{Arthur Rosenheim und A. Garfunkel: Zur Kenntnis der Kobaltinitrite.}

(Eingeg. am 20. Juni 1911; vorgetragen in der Sitzung von Hrn. A. Rosen heim.)

Gelegentlich einer eingehenden Untersuchung der komplexen Kobaltinitrite fanden A. Rosenheim und I. Koppel ${ }^{1}$ ) eine Reihe von Salzen, die weniger als sechs Nitritgruppen im komplexen Anion enthielten. $\mathrm{Da}$ in Bestätigung der Wernerschen Theorie alle bisher untersuchten Komplexverbindungen des dreiwertigen Kobalts sechs Gruppen um das Zentralatom koordinierta enthalten, so muBten in diesen Anionen Sauerstoffatome, Hydroxylgruppen oder Wassermoleküle die an der Zahl sechs fehlenden Nitritgruppen ersetzen. Die Untersuchung solcher bydroxylhaltigen komplexen Anionen, von denen bisher nur wenige bekannt sind \%), ist für die Kenntnis der Komplexverbindungen von wesentlichem Interesse.

l) Z. a. Ch. 17, 35 [1898].

3) Vergl. Z. a. Ch. 65, 173 [1909]. 